\title{
Second dielectric virial coefficient of helium gas: quantum-statistical calculations from an ab initio interaction-induced polarizability
}

\author{
Robert Moszynski ${ }^{\text {a,b }}$, Tino G.A. Heijmen ${ }^{a}$, Ad van der Avoird ${ }^{\text {a }}$ \\ anstitute of Theoretical Chemistry, Nijmegen - SON Research Center, University of Nijmegen, Toernooiveld 1, 6525 ED Nijmegen, \\ The Netherlands \\ ${ }^{\mathrm{b}}$ Department of Chemistry, University of Warsaw, Pasteura 1,02-093 Warsaw, Poland
}

Received 10 July 1995; in final form 27 October 1995

\begin{abstract}
A quantum-statistical expression for the second virial coefficient of the dielectric Clausius-Mossotti function is derived. Calculations of the second dielectric virial coefficient of helium gas at various temperatures are reported. The role of quantum mechanical effects and the convergence properties of the semiclassical expansion are discussed. The calculated second dielectric virial coefficients at various temperatures are compared with experimental data.
\end{abstract}

\section{Introduction}

It is well known that for atomic gases at low densities the Clausius-Mossotti function can be related to the atomic polarizability via the relation

$\frac{\epsilon-1}{\epsilon+2}=\frac{4 \pi \alpha_{0}}{3} \rho$

where $\epsilon$ is the dielectric constant, $\alpha_{0}$ is the atomic polarizability, and $\rho$ denotes the gas number density. At higher pressures deviations from this relation are observed, and they can be attributed to intermolecular interactions. Buckingham and Pople [1] have shown that the leading correction to Eq. (1), quadratic in the gas density, is given by $B_{\epsilon}(T) \rho^{2}$, where at high temperatures the second dielectric virial coeffi- cient $B_{\epsilon}(T)$ is related to the interatomic potential and interaction-induced polarizability by

$B_{\epsilon}(T)=\frac{2 \pi}{3} \int \Delta \alpha(R) \exp \left[-V(R) / k_{\mathrm{B}} T\right] \mathrm{d} \boldsymbol{R}$.

Here $T$ is the temperature, $k_{\mathrm{B}}$ is the Boltzmann constant, $V(R)$ is the pair interaction potential, and $\Delta \alpha(R)$ is the trace of the interaction-induced polarizability tensor.

Much progress has been made in the development of various experimental techniques for measuring the second dielectric virial coefficients, and at present data are available for various atomic and molecular systems (see Refs. [2,3] for recent reviews of experimental data). The dielectric properties of helium gas are of particular interest, since for this system accu- 
rate theoretical data can be obtained. Consequently, since the early 1960 s more and more accurate measurements [4-13] have been reported in the literature. Low-temperature results $[10,11]$ are especially interesting since they can be compared to theoretical investigations of the quantum effects on the second dielectric virial coefficient.

While classical calculations of the second dielectric virial coefficient for helium have been reported by several authors (see Ref. [2] for a review), the importance of the quantum corrections has been the object of only a few studies [14-17]. The semiclassical expansion of the second dielectric virial coefficient as a power series in $\hbar^{2}$ has been considered in Refs. [16,17]. Ely and McQuarrie [16] derived an expression for the leading quantum correction to the classical term of Eq. (1), and applied it in calculations for a Lennard-Jones gas with the interaction-induced polarizability trace from the simple dipole-induced-dipole model. They concluded that at low temperatures the first quantum correction was important and represents as much as $53 \%$ and $25 \%$ of the classical term at $T=15.33$ and $25.55 \mathrm{~K}$, respectively. Approximate quantum calculations have been reported by Bruch et al. [17]. Unfortunately, these authors restricted their work to a single temperature, and did not compare the quantum results with the (semi)classical results.

In a recent paper [18] we reported symmetryadapted perturbation theory (SAPT) calculations (see Ref. [19] for a recent review of SAPT and its applications) of the interaction-induced polarizability for the $\mathrm{He}$ diatom, and dynamical calculations of the polarized and depolarized Raman intensities. We also checked the ab initio collision-induced polarizability by computation of the semiclassical approximations to the second dielectric virial coefficient, and by comparison with experiment. Our results suggested that at low temperatures the semiclassical expressions for $B_{\epsilon}(T)$ are no longer valid. Hence, it seems that a more systematic study of the quantum effects on the second dielectric virial coefficients is needed.

A general expression relating the second dielectric virial coefficient in an electric field has been reported by Hill [20] (see also Ref. [21]). Unfortunately, Hill introduced some simplifying assumptions in his derivation, and at high temperatures his for- mula for $B_{\epsilon}(T)$ does not reduce to the classical result of Buckingham and Pople [1], cf. Eq. (2). Thus, the expression of Ref. [20] must be modified before it could be used to derive the quantum equivalent of Eq. (2).

In this Letter we report the derivation of the quantum expression for the second dielectric virial coefficient, and ab initio calculations of $B_{\epsilon}(T)$ for $\mathrm{He}$ at various temperatures. The results of the exact quantum-statistical calculations are compared with the semiclassical approximations including first and second quantum corrections, as well as with the experimental data.

\section{Theory}

We follow Hill [20] and consider an atomic gas in a parallel plate capacitor with surface charges which yield in the vacuum a uniform electric field $\boldsymbol{F}$. For simplicity we assume the $z$ direction to be parallel to the field, so $\boldsymbol{F}=(0,0, F)$. As shown in Ref. [20], the polarization $P$ along the electric field vector $\boldsymbol{F}$ can be written as a power series expansion in the gas density $\rho$,

$P=P_{1} V \rho-k_{\mathrm{B}} T \sum_{n=2} \frac{1}{n-1}\left(\frac{\partial B_{n}(T ; F)}{\partial F}\right) \rho^{n}$,

where $P_{1}$ is the polarization of a single atom, cf. Eq. (6), $V$ is the volume, and $B_{n}(T ; F)$ denotes ordinary (pressure) virial coefficients for the gas in the electric field $F$. Since we are interested in the low field limit. Eq. (3) can conveniently be rewritten as

$$
\begin{aligned}
P= & P_{1} V \rho-k_{\mathrm{B}} T \sum_{n=2} \frac{1}{n-1}\left(\frac{\partial^{2} B_{n}(T ; F)}{\partial F^{2}}\right)_{F=0} F \rho^{n} \\
& +O\left(F^{3}\right) .
\end{aligned}
$$

When deriving Eq. (4) we have used the fact that $B_{n}(T ; F)$ is an even function of $F$, so

$$
\frac{\partial B_{n}(T ; F)}{\partial F}=\left(\frac{\partial^{2} B_{n}(T ; F)}{\partial F^{2}}\right)_{F=0} F+O\left(F^{3}\right) \text {. }
$$

Furthermore, from the Lorentz equation [22] we have

$$
P_{1} V=\alpha_{0} F_{\text {loc }}=\alpha_{0} \frac{\epsilon+2}{3} F,
$$


where $F_{\text {loc }}$ is the local electric field which actually polarizes an atom or the assembly of atoms in the gas. Note that at this point our derivation differs from that of Hill [20]. In the latter it was assumed that $P_{1} V$ is proportional to the field $F$ with the proportionality constant $\alpha_{1}(T)$ approximated by $\alpha_{0}$, or equivalently, the local field $F_{\text {loc }}$ was approximated by the field $F$. Substituting for $P$,

$P=\frac{\epsilon-1}{4 \pi} F$,

we obtain

$$
\begin{aligned}
\epsilon-1= & (\epsilon+2) \frac{4 \pi \alpha_{0}}{3} \rho-4 \pi k_{\mathrm{B}} T \\
& \times \sum_{n=2} \frac{1}{n-1}\left(\frac{\partial^{2} B_{n}(T ; F)}{\partial F^{2}}\right)_{F=0} \rho^{n} .
\end{aligned}
$$

Eq. (8) can be transformed to a power series expansion of the Clausius-Mossotti function,

$$
\frac{\epsilon-1}{\epsilon+2}=A_{\epsilon} \rho+B_{\epsilon}(T) \rho^{2}+C_{\epsilon}(T) \rho^{3}+\ldots,
$$

where

$A_{\epsilon}=\frac{4 \pi \alpha_{0}}{3}$,

$B_{\epsilon}(T)=\frac{4 \pi k_{\mathrm{B}} T}{3}\left(\frac{\partial^{2} B_{2}(T ; F)}{\partial F^{2}}\right)_{F=0}$,

$$
\begin{aligned}
C_{\epsilon}(T)= & -2 A_{\epsilon} B_{\epsilon}(T) \\
& -\frac{2 \pi k_{\mathrm{B}} T}{3}\left(\frac{\partial^{2} B_{3}(T ; F)}{\partial F^{2}}\right)_{F=0} .
\end{aligned}
$$

Our expression for $B_{\epsilon}(T)$ differs from the formula derived by Hill [20], and it is not difficult to show that at high temperatures Eq. (11) reduces to the classical expression, Eq. (2). Our Eq. (11) can be obtained from his Eq. (33) by assuming that $\alpha_{1}(\mathrm{~T})$ is not approximated by the polarizability of the atom, but is instead given by a power series expansion in $\rho$.

The field-dependent second virial coefficient for an atomic gas can conveniently be written as [23]

$$
B_{2}(T ; F)=\frac{1}{2} \int[W(\boldsymbol{R} ; F)-1] \mathrm{d} \boldsymbol{R} .
$$

where $W(\boldsymbol{R} ; F)$ denotes the Slater sum

$$
\begin{aligned}
W(\boldsymbol{R} ; F)= & \lambda_{\mathrm{B}}^{3}\left\langle\boldsymbol{R}\left|\mathrm{e}^{-\beta H(F)}\right| \boldsymbol{R}\right\rangle \\
& +\frac{\lambda_{\mathrm{B}}^{3}(-1)^{2 I}}{2 I+1}\left\langle-\boldsymbol{R}\left|\mathrm{e}^{-\beta H(F)}\right| \boldsymbol{R}\right\rangle .
\end{aligned}
$$

Here, $\lambda_{\mathrm{B}}=\left(4 \pi \hbar^{2} / k_{\mathrm{B}} T m\right)^{1 / 2}$ is the thermal wavelength, $m$ is the atomic mass, $\beta=1 / k_{\mathrm{B}} T, l$ is the nuclear spin quantum number, and $\left\langle\boldsymbol{R}^{\prime}|X| \boldsymbol{R}\right\rangle$ denotes the matrix element of the operator $X$ in the coordinate representation. Finally, the Hamiltonian for the relative motion of two atoms in the electric field is given by

$$
\begin{gathered}
H(F)=H_{0}-\frac{1}{2} \Delta \alpha_{z z}(R) F^{2}, \\
H_{0}=-\frac{\hbar^{2}}{m} \nabla^{2}+V(R),
\end{gathered}
$$

where $V(R)$ is the pair interaction potential, and $\Delta \alpha_{z z}(R)$ denotes the $z z$ component of the interaction-induced polarizability. For further discussion it is useful to write $\Delta \alpha_{z z}(R)$ as

$\Delta \alpha_{z z}(\boldsymbol{R})=\Delta \alpha(R)+\frac{2}{3} \gamma(R) P_{2}(\cos \vartheta)$,

where $\Delta \alpha(R)$ and $\gamma(R)$ denote the interaction-induced trace and anisotropy of the polarizability tensor, $P_{2}$ is the second Legendre polynomial, and $\vartheta$ is the angle between the $z$ axis and the dimer axis.

To derive a quantum expression for $B_{\epsilon}(T)$ we need the second derivative of the Slater sum in the electric field. Using simple algebraic arguments [24] one can show that

$$
\begin{aligned}
& \left(\frac{\partial^{2}}{\partial F^{2}}\left\langle\boldsymbol{R}^{\prime}\left|\mathrm{e}^{-\beta H(F)}\right| \boldsymbol{R}\right\rangle\right)_{F=0} \\
& \quad=\beta\left\langle\boldsymbol{R}^{\prime}\left|\exp \left(-\beta H_{0}\right) \Delta \alpha_{z z}(\boldsymbol{R})\right| \boldsymbol{R}\right\rangle .
\end{aligned}
$$

Note that $\Delta \alpha_{z z}(\boldsymbol{R})$ is diagonal in $\boldsymbol{R}$, so expression (17) can be further simplified to

$$
\begin{aligned}
& \left\langle\boldsymbol{R}^{\prime}\left|\exp \left(-\beta H_{0}\right) \Delta \alpha_{z z}(\boldsymbol{R})\right| \boldsymbol{R}\right\rangle \\
& \quad=\Delta \alpha_{z z}(\boldsymbol{R})\left\langle\boldsymbol{R}^{\prime}\left|\exp \left(-\beta H_{0}\right)\right| \boldsymbol{R}\right\rangle .
\end{aligned}
$$

In view of Eqs. (11), (13), (17), and (18), the expression for $B_{\epsilon}(T)$ takes the form,

$$
B_{\epsilon}(T)=\frac{2 \pi}{3} \int W(\boldsymbol{R} ; 0) \Delta \alpha_{z z}(\boldsymbol{R}) \mathrm{d} \boldsymbol{R} .
$$


A similar formula has been reported by Bruch et al. [17]. One may note that in practical applications the evaluation of this expression requires knowledge of the Slater sum at zero field.

Eq. (19) can be further simplified by introducing a complete set of (bound and continuum) eigenfunctions of the Hamitonian $H_{0}$,

$H_{0} \Psi_{n J M}=E_{n J} \Psi_{n J M}$,

$H_{0} \Psi_{k J M}=\frac{\hbar^{2} k^{2}}{m} \Psi_{k J M}$,

where $E_{n J}$ and $E_{k} \equiv \hbar^{2} k^{2} / m$ are the eigenvalues of bound and continuum states, respectively, and the corresponding wavefunctions are given by

$\Psi_{n J M}(R)=R^{-1} \chi_{n J}(R) Y_{M}^{J}(\vartheta, \varphi)$.

$\Psi_{k J M}(R)=\left(\frac{2}{\pi}\right)^{1 / 2} R^{-1} \chi_{k J}(R) Y_{M}^{J}(\vartheta, \varphi)$

where $Y_{M}^{J}(\vartheta, \varphi)$ are the normalized spherical harmonics. We assume that the radial wave-functions are solutions of the radial Schrödinger equation describing the relative motion of the atoms in the potential $V(R)$,

$-\frac{\hbar^{2}}{m} \frac{\mathrm{d}^{2} \chi}{\mathrm{d} R^{2}}+\left(V(R)+\frac{\hbar^{2} J(J+1)}{m R^{2}}-E\right) \chi=0$,

subject to the following normalization conditions,

$\int_{0}^{\infty} \chi_{n J}^{*}(R) \chi_{n^{\prime} J}(R) \mathrm{d} R=\delta_{n n^{\prime}}$,

$\int_{0}^{\infty} \chi_{k^{\prime} J}^{*}(R) \chi_{k^{\prime} J}(R) \mathrm{d} R=\delta\left(E_{\boldsymbol{k}}-E_{\boldsymbol{k}^{\prime}}\right)$.

The expression for $W(\boldsymbol{R} ; 0)$ can conveniently be written as a sum-over-states formula [25],

$W(\boldsymbol{R} ; 0)$

$$
\begin{aligned}
= & \frac{\lambda_{\mathrm{B}}^{3}}{4 \pi} \sum_{J=0}^{\infty}(2 J+1)\left(1+\frac{(-1)^{J+2 I}}{2 I+1}\right) \\
& \times\left(\sum_{n} \exp \left(-\beta E_{n J}\right) \chi_{n J}^{*}(R) \chi_{n J}(R)\right. \\
+ & \left.\frac{2}{\pi} \int_{0}^{\infty} \exp \left(-\beta \hbar^{2} k^{2} / m\right) \chi_{k J}^{*}(R) \chi_{k J}(R) \mathrm{d} k\right) .
\end{aligned}
$$

By substituting into Eq. (19) the expression for $W(\boldsymbol{R} ; 0)$ in terms of the complete set of eigenfunctions of $H_{0}$ one finds that $B_{\epsilon}(T)$ is given by

$$
\begin{aligned}
B_{\epsilon}(T)= & \frac{2 \pi \lambda_{\mathrm{B}}^{3}}{3} \sum_{J=0}^{\infty}(2 J+1)\left(1+\frac{(-1)^{J+2 I}}{2 I+1}\right) \\
& \times\left(\sum_{n} \exp \left(-\beta E_{n J}\right)\right. \\
& \times\left\langle\chi_{n J}(R)|\Delta \alpha(R)| \chi_{n J}(R)\right\rangle \\
& +\frac{2}{\pi} \int_{0}^{\infty} \exp \left(-\beta \hbar^{2} k^{2} / m\right) \\
& \left.\times\left\langle\chi_{k J}(R)|\Delta \alpha(R)| \chi_{k J}(R)\right\rangle \mathrm{d} k\right),
\end{aligned}
$$

where the matrix elements of the trace appearing in Eq. (26) are defined as

$$
\langle\chi|\Delta \alpha(R)| \chi\rangle=\int_{0}^{\infty} \chi^{*}(R) \Delta \alpha(R) \chi(R) \mathrm{d} R .
$$

Using our ab initio interaction-induced trace data [18] (see Fig. 1 for a graphical illustration of the dependence of $\Delta \alpha(R)$ on the interatomic distance $R$ ) and the empirical interaction potential [26] we have computed the second dielectric virial coefficient over a wide range of temperatures. The

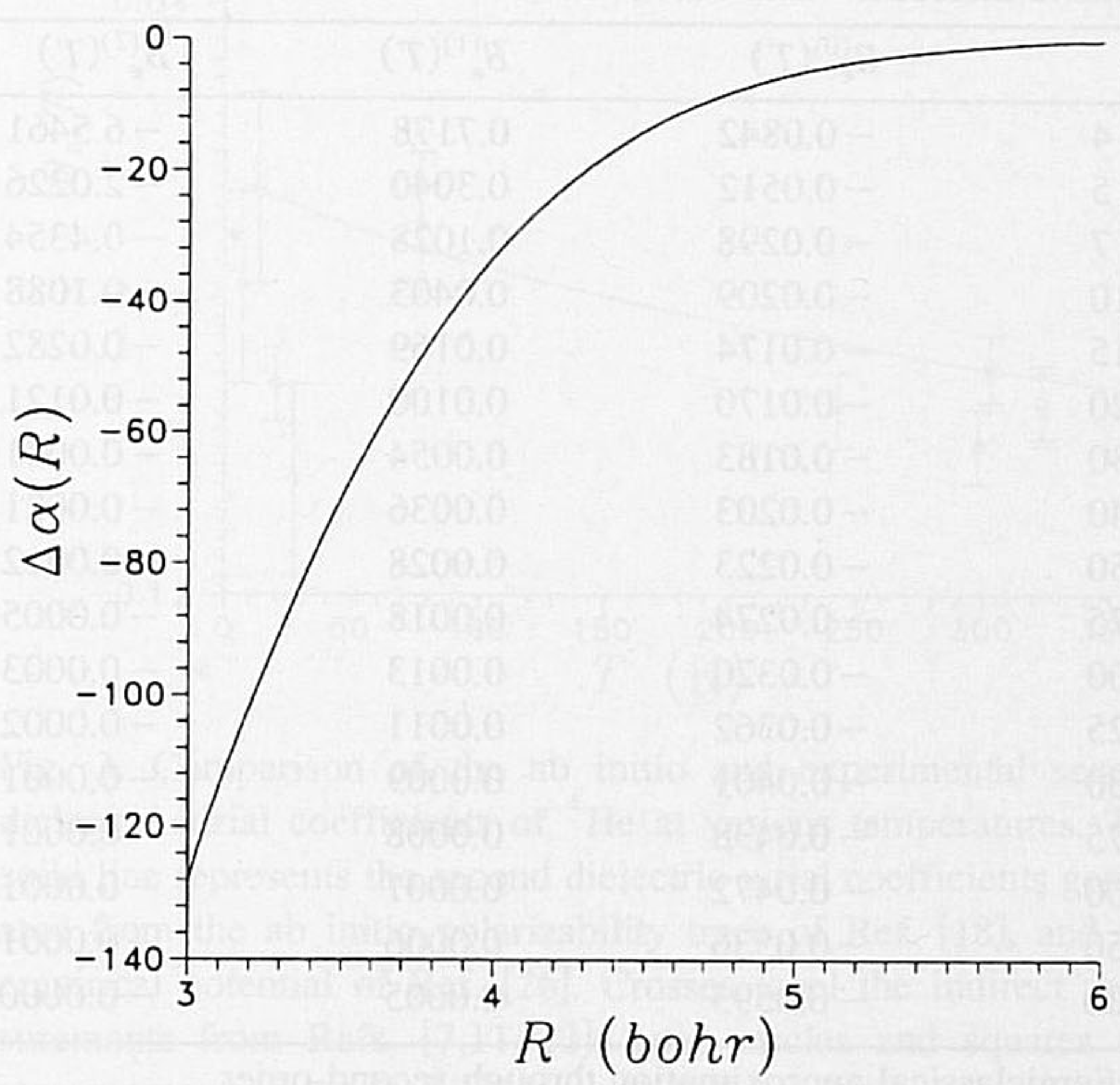

Fig. 1. Trace polarizability of $\mathrm{He}_{2}$ (in $10^{-3}$ au) as a function of the interatomic separation $R$ (in bohr). 
Schrödinger equation (23) was solved using the $\mathrm{Nu}$ merov method. The calculations were done with the BCONT program of Le Roy $[27,28]$ adapted for the present purposes. The $k$-dependent matrix elements were obtained at a fine mesh of 1000 values of the wavevector. The thermal averaging was done using an extended Simpson rule on the interval from $k_{\min }$ $=0.01$ to $k_{\text {mid }}=1.5 \mathrm{au}$, combined with a cubic spline integration over $k$ between $k_{\text {mid }}$ and $k_{\max }=16$ au. The integration parameters, and the number of partial wave components, were chosen to yield second dielectric virial coefficients converged to $2 \%$ at worst. The mass of the ${ }^{4} \mathrm{He}$ atom was fixed at $4.00260 \mathrm{amu}$ [29].

\section{Numerical results and discussion}

The results of quantum statistical calculations of $B_{\epsilon}(T)$ as a function of temperature are presented in Table 1. The main contribution to the second dielectric virial coefficient of ${ }^{4} \mathrm{He}$ gas comes from the summation over the continuum in Eq. (26). The bound state contribution is always small, and even at the lowest temperatures it does not exceed $2 \%$. Also presented in this table is the classical dielectric virial coefficient computed with Eq. (2) (denoted by
$B_{\epsilon}^{(0)}(T)$ ), and the first and second quantum corrections (denoted by $B_{\epsilon}^{(1)}(T)$ and $B_{\epsilon}^{(2)}(T)$, respectively). The quantum corrections have been computed from the expressions reported in Ref. [18]. These formulas were derived by applying Eq. (11) to the semiclassical expansion of the ordinary (pressure) virial coefficient in an electric field [30]. An inspection of Table 1 shows that the quantum effects are small for temperatures larger than $100 \mathrm{~K}$, and $B_{\epsilon}(T)$ can be approximated by the classical expression with an error smaller than $2.5 \%$. At lower temperatures the dielectric virial coefficient of ${ }^{4} \mathrm{He}$ starts to deviate from the classical value. Still, for $T \geqslant 50 \mathrm{~K}$ the quantum effects can be efficiently accounted for by the sum of the first and second quantum corrections. Indeed, for $T=50,75$, and $100 \mathrm{~K}$ the series $B_{\epsilon}^{(0)}(T)$ $+B_{\epsilon}^{(1)}(T)+B_{\epsilon}^{(2)}(T)$ reproduces the exact results with errors smaller than $2 \%$. One may note that at these temperatures the second quantum correction is small, and can be neglected for all practical purposes. In fact, the sum $B_{\epsilon}^{(0)}(T)+B_{\epsilon}^{(1)}(T)$ slightly overestimates the exact result, while $B_{\epsilon}^{(0)}(T)+B_{\epsilon}^{(1)}(T)+$ $B_{\epsilon}^{(2)}(T)$ slightly underestimates it. At temperatures below $50 \mathrm{~K}$ the semiclassical expansion of the second dielectric virial coefficient in powers of $\hbar^{2}$ starts to diverge. This divergence is clearly illustrated in Fig. 2, where various approximations to $B_{\epsilon}(T)$ are

Table 1

Second dielectric virial coefficient of ${ }^{4} \mathrm{He}\left(\right.$ in $\mathrm{cm}^{6} \mathrm{~mol}^{-2}$ ) as a function of temperature (in $\mathrm{K}$ )

\begin{tabular}{rllllll}
\hline$T$ & $B_{\epsilon}^{(0)}(T)$ & $B_{\epsilon}^{(1)}(T)$ & $B_{\epsilon}^{(2)}(T)$ & $B_{\epsilon}(T)^{\mathrm{a}}$ & {$[1 / 1]^{\mathrm{b}}$} & $B_{\epsilon}(T)^{\mathrm{c}}$ \\
\hline 4 & -0.0842 & 0.7178 & -6.5461 & -5.9125 & -0.0133 & -0.0081 \\
5 & -0.0512 & 0.3040 & -2.0226 & -1.7699 & -0.0115 & -0.0081 \\
7 & -0.0298 & 0.1028 & -0.4354 & -0.3624 & -0.0102 & -0.0085 \\
10 & -0.0209 & 0.0403 & -0.1088 & -0.0894 & -0.0100 & -0.0093 \\
15 & -0.0174 & 0.0169 & -0.0282 & -0.0287 & -0.0111 & -0.0108 \\
20 & -0.0170 & 0.0100 & -0.0121 & -0.0191 & -0.0125 & -0.0121 \\
30 & -0.0183 & 0.0054 & -0.0041 & -0.0171 & -0.0153 & -0.0152 \\
40 & -0.0203 & 0.0036 & -0.0021 & -0.0187 & -0.0179 & -0.0179 \\
50 & -0.0223 & 0.0028 & -0.0012 & -0.0208 & -0.0204 & -0.0204 \\
75 & -0.0274 & 0.0018 & -0.0005 & -0.0261 & -0.0260 & -0.0260 \\
100 & -0.0320 & 0.0013 & -0.0003 & -0.0309 & -0.0309 & -0.0309 \\
125 & -0.0362 & 0.0011 & -0.0002 & -0.0353 & -0.0353 & -0.0353 \\
150 & -0.0401 & 0.0009 & -0.0001 & -0.0393 & -0.0393 & -0.0393 \\
175 & -0.0438 & 0.0008 & -0.0001 & -0.0431 & -0.0431 & -0.0431 \\
200 & -0.0472 & 0.0007 & -0.0001 & -0.0466 & -0.0466 & -0.0466 \\
250 & -0.0536 & 0.0006 & -0.0001 & -0.0530 & -0.0530 & -0.0530 \\
300 & -0.0593 & 0.0005 & -0.0000 & -0.0588 & -0.0588 & -0.0588 \\
\hline
\end{tabular}

\footnotetext{
a Semiclassical approximation through second-order.

b Padé approximant applied to the series $B_{\epsilon}^{(0)}(T)+B_{\epsilon}^{(1)}(T)+B_{\epsilon}^{(2)}(T)$.

${ }^{c}$ Exact quantum-statistical calculation.
} 


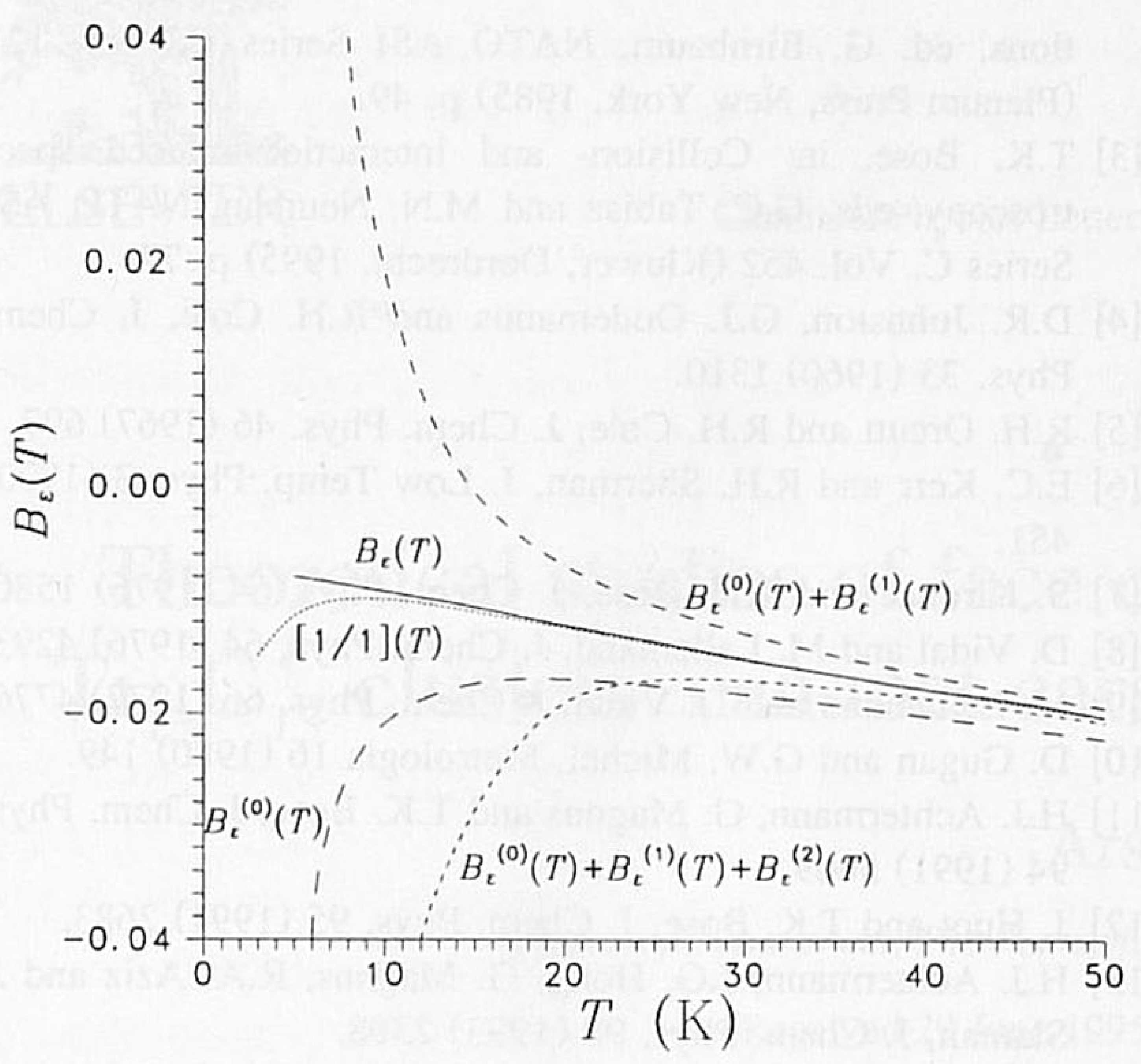

Fig. 2. Temperature dependence of the second dielectric virial coefficient (in $\mathrm{cm}^{6} \mathrm{~mol}^{-2}$ ) of ${ }^{4} \mathrm{He}$ gas.

plotted as a function of the temperature. This behaviour of the power series in $\hbar^{2}$ is not surprising, since the semiclassical expansion of the pressure virial coefficient is known to diverge as well (see, e.g., Ref. [31]).

Given the overall pattern of convergence of the semiclassical expansion, it is interesting to find whether any rational approximations involving the low-order quantum corrections will reproduce the converged quantum result. It is well known [32] that divergent series can be effectively summed by means of Padé approximants. Since we know only three terms in the expansion of $B_{\epsilon}(T)$ as a power series in $\hbar^{2}$, we could only use the simplest [1/1] approximant. The values of this approximant at various temperatures are reported in the sixth column of Table 1. Except for the lowest temperatures, the simple [1/1] Padé approximant works surprisingly well. For $T=15$ and $20 \mathrm{~K}$ the sum of the classical term and first and second quantum corrections overestimates the exact result by $265 \%$ and $58 \%$, respectively, while the [1/1] approximant reproduces the quantum results with errors of the order of $3 \%$. This result is gratifying since the calculation of the quantum corrections is much simpler than full quantumstatistical calculations. It remains to be seen, however, whether this optimistic result holds for other systems as well.
Table 2

Comparison of the computed and measured second dielectric virial coefficients of ${ }^{4} \mathrm{He}$ (in $\mathrm{cm}^{6} \mathrm{~mol}^{-2}$ ) at various temperatures (in $\mathrm{K}$ )

\begin{tabular}{llllll}
\hline$T$ & $B_{\epsilon}(T)$ & $\Delta^{\mathrm{a}}$ & Exp. & $\Delta^{\mathrm{b}}$ & Ref. \\
\hline 3.799 & -0.0081 & \pm 0.0004 & -0.023 & $?$ & {$[6]$} \\
4.220 & -0.0081 & \pm 0.0004 & 0.083 & \pm 0.026 & {$[10]$} \\
7.199 & -0.0092 & \pm 0.0004 & -0.036 & \pm 0.026 & {$[10]$} \\
13.804 & -0.0104 & \pm 0.0005 & -0.010 & \pm 0.026 & {$[10]$} \\
20.271 & -0.0123 & \pm 0.0006 & -0.062 & \pm 0.010 & {$[10]$} \\
27.098 & -0.0144 & \pm 0.0007 & -0.088 & \pm 0.026 & {$[10]$} \\
77.4 & -0.0265 & \pm 0.0013 & -0.02 & \pm 0.02 & {$[12]$} \\
242.95 & -0.0522 & \pm 0.0026 & -0.07 & \pm 0.01 & {$[12]$} \\
298.15 & -0.0587 & \pm 0.0029 & -0.11 & \pm 0.02 & {$[8]$} \\
303 & & & -0.08 & \pm 0.01 & {$[9]$} \\
& -0.0592 & \pm 0.0031 & -0.059 & \pm 0.009 & {$[7]$} \\
322.15 & -0.0613 & \pm 0.0031 & -0.06 & \pm 0.04 & {$[5]$} \\
323 & -0.0614 & \pm 0.0031 & -0.068 & \pm 0.01 & {$[11]$} \\
323.15 & -0.0614 & \pm 0.0031 & -0.07 & \pm 0.01 & {$[12]$} \\
\hline
\end{tabular}

"Theoretical error bars assuming the interaction-induced polarizability trace accurate to $\pm 5 \%$.

${ }^{b}$ Experimental error bars.

In Table 2 and Fig. 3 we compare the computed second dielectric virial coefficients of ${ }^{4} \mathrm{He}$ with experimental data [5-13]. In Ref. [18] we have care-

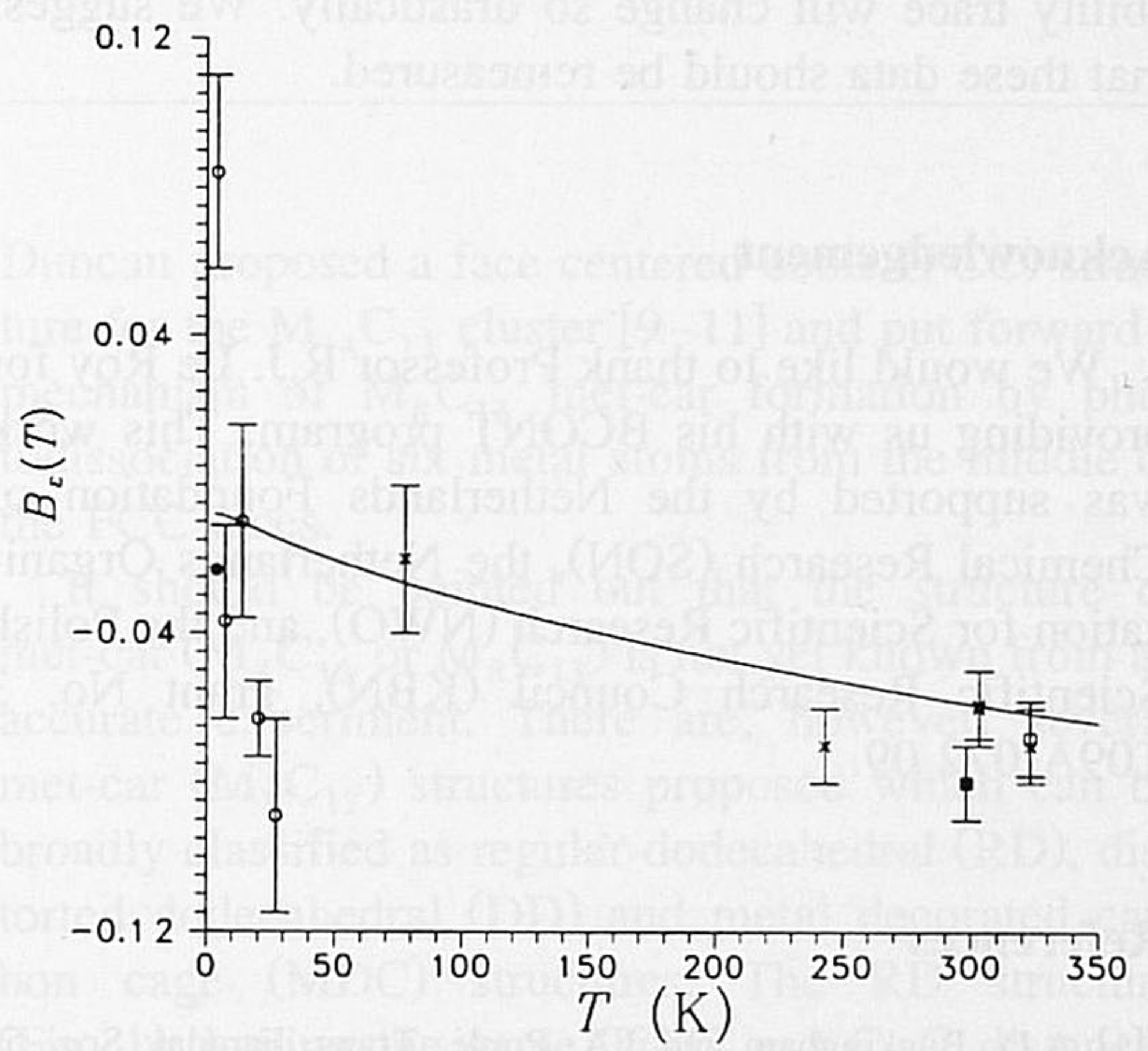

Fig. 3. Comparison of the ab initio and experimental second dielectric virial coefficients of ${ }^{4} \mathrm{He}$ at various temperatures. The solid line represents the second dielectric virial coefficients generated from the ab initio polarizability trace of Ref. [18], and the empirical potential of Ref. [26]. Crosses label the indirect measurements from Refs. [7,11-13], open circles and squares the measurements from Refs. [10] and [15], respectively, and filled circles and squares represent experimental data from Refs. [6] and $[8,9]$. 
fully checked the convergence of the SAPT expansion by comparison with the exact results from full configuration interaction calculations, as well as the basis-set convergence. We have estimated the residual error in our polarizability trace to be $5 \%$ at worst. Since the second dielectric virial coefficient is linear in $\Delta \alpha$ our values for $B_{\epsilon}(T)$ should be accurate to $5 \%$ or better. At high temperatures our results agree well with the data from indirect measurements [7,11-13]. The only exception is the value at $T=$ $242.95 \mathrm{~K}$. Here the difference is as large as $25 \%$. Our results suggest that the second dielectric virial coefficient at this temperature should be larger than $-0.55 \mathrm{~cm}^{6} \mathrm{~mol}^{-2}$.

At low temperatures the situation is more complex. Our result at $77.4 \mathrm{~K}$ agrees well with the value from indirect measurements reported by Huot and Bose [12]. Other low temperature data were obtained from direct measurements [10] and show a lot of scatter. At $T=13.804 \mathrm{~K}$ our value agrees with the measurement, and at $T=7.198 \mathrm{~K}$ the theoretical result is almost within the experimental error bars. At other temperatures the disagreement is quite substantial, and it is unlikely that the theoretical polarizability trace will change so drastically. We suggest that these data should be remeasured.

\section{Acknowledgement}

We would like to thank Professor R.J. Le Roy for providing us with his BCONT program. This work was supported by the Netherlands Foundation of Chemical Research (SON), the Netherlands Organization for Scientific Research (NWO), and the Polish Scientific Research Council (KBN), grant No. 3 T09A 07209.

\section{References}

[1] A.D. Buckingham and J.A. Pople, Trans. Faraday Soc. 51 (1955) 1029, 1079.

[2] T.K. Bose, in: Phenomena induced by intermolecular interac- tions, ed. G. Birnbaum, NATO ASI Series B, Vol. 127 (Plenum Press, New York, 1985) p. 49.

[3] T.K. Bose, in: Collision- and interaction-induced spectroscopy, eds. G.C. Tabisz and M.N. Neuman, NATO ASI Series C, Vol. 452 (Kluwer, Dordrecht, 1995) p. 77.

[4] D.R. Johnston, G.J. Oudemanns and R.H. Cole, J. Chem. Phys. 33 (1960) 1310.

[5] R.H. Orcutt and R.H. Cole, J. Chem. Phys. 46 (1967) 697.

[6] E.C. Kerr and R.H. Sherman, J. Low Temp. Phys. 3 (1970) 451.

[7] S. Kirouac and T.K. Bose, J. Chem. Phys. 64 (1976) 1580.

[8] D. Vidal and M. Lallemand, J. Chem. Phys. 64 (1976) 4293.

[9] M. Lallemand and D. Vidal, J. Chem. Phys. 66 (1977) 4776.

[10] D. Gugan and G.W. Michel, Metrologia 16 (1980) 149.

[11] H.J. Achtermann, G. Magnus and T.K. Bose, J. Chem. Phys. 94 (1991) 5669.

[12] J. Huot and T.K. Bose, J. Chem. Phys. 95 (1991) 2683.

[13] H.J. Achtermann, J.G. Hong, G. Magnus, R.A. Aziz and J. Slaman, J. Chem. Phys. 98 (1993) 2308.

[14] A. Isihara and R.V. Hanks, J. Chem. Phys. 36 (1962) 433; A. Isihara, J. Chem. Phys. 38 (1963) 2437.

[15] D.A. McQuarrie and H.B. Levine, Physica 31 (1965) 749.

[16] J.F. Ely and D.A. McQuarrie, J. Chem. Phys. 54 (1971) 2885.

[17] L.W. Bruch, P.J. Fortune and D.H. Berman, J. Chem. Phys. 61 (1974) 2626.

[18] R. Moszynski, T.G.A. Heijmen, P.E.S. Wormer and A. van der Avoird, submitted for publication.

[19] B. Jeziorski, R. Moszynski and K. Szalewicz, Chem. Rev. 94 (1994) 1887.

[20] T.L. Hill, J. Chem. Phys. 28 (1958) 61.

[21] A.D. Buckingham and R.E. Raab, Trans. Faraday Soc. 54 (1958) 623.

[22] H. Fröhlich, Theory of dielectrics, dielectric constant, and dielectric loss (Clarendon Press, Oxford, 1963).

[23] J. de Boer, Rept. Prog. Phys. 12 (1949) 305.

[24] H. Falk, Phys. Rev. 165 (1968) 602.

[25] J.M. Blatt, Nuovo Cimento 4 (1956) 430.

[26] R.A. Aziz, F.R. McCourt and C.C.K. Wong, Mol. Phys. 61 (1987) 1487.

[27] R.J. Le Roy, Comput. Phys. Commun. 52 (1989) 383.

[28] R.J. Le Roy, University of Waterloo Chemical Physics Research Report CP-329R (1993).

[29] R.C. Weast and M.J. Asth, eds. Handbook of chemistry and physics (Chemical Rubber, Boca Raton, 1981).

[30] J.O. Hirschfelder, C.F. Curtiss and R.B. Bird, Molecular theory of gases and liquids (Wiley, New York, 1964).

[31] D. ter Haar, Elements of statistical mechanics (Reinehart, New York, 1964).

[32] B. Jeziorski, W.A. Schwalm and K. Szalewicz, J. Chem. Phys. 73 (1980) 6215. 